\title{
Emergence of running dark energy from polynomial $f(R)$ theory in Palatini formalism
}

\author{
Marek Szydłowski $^{1,2, a}$, Aleksander Stachowski ${ }^{1, b}$, Andrzej Borowiec ${ }^{3, c}$ \\ ${ }^{1}$ Astronomical Observatory, Jagiellonian University, Orla 171, 30-244 Kraków, Poland \\ ${ }^{2}$ Mark Kac Complex Systems Research Centre, Jagiellonian University, ul. Łojasiewicza 11, 30-348 Kraków, Poland \\ ${ }^{3}$ Institute for Theoretical Physics, University of Wrocław, pl. M. Borna 9, 50-204 Wrocław, Poland
}

Received: 25 July 2017 / Accepted: 30 August 2017 / Published online: 12 September 2017

(C) The Author(s) 2017. This article is an open access publication

\begin{abstract}
We consider FRW cosmology in $f(R)=R+$ $\gamma R^{2}+\delta R^{3}$ modified framework. The Palatini approach reduces its dynamics to the simple generalization of Friedmann equation. Thus we study the dynamics in twodimensional phase space with some details. After reformulation of the model in the Einstein frame, it reduces to the FRW cosmological model with a homogeneous scalar field and vanishing kinetic energy term. This potential determines the running cosmological constant term as a function of the Ricci scalar. As a result we obtain the emergent dark energy parametrization from the covariant theory. We study also singularities of the model and demonstrate that in the Einstein frame some undesirable singularities disappear.
\end{abstract}

\section{Introduction}

A variety of explanations have been proposed for the accelerating expansion of the universe at the current epoch. Among them, the idea of positive cosmological constant $\Lambda$, as one of the simplest candidates, seems to be viable. However, it is only an economical description (with the help of one free parameter) of observational facts rather than an effective explanation. The simplest alternative candidate for the constant cosmological parameter being a key element in the standard cosmological model (called $\Lambda$ CDM model) is a time-dependent (or running) cosmological term. It is crucial for avoiding fine-tuning and coincidence problems [1,2].

It would be crucial to derive the dynamics of the running cosmological term as an emergent phenomenon from a more fundamental theory, e.g., from string theory or from the first principles of quantum mechanics [3]. In this context,

\footnotetext{
${ }^{a}$ e-mail: marek.szydlowski@uj.edu.pl

be-mail: aleksander.stachowski@doctoral.uj.edu.pl

c e-mail: andrzej.borowiec@ift.uni.wroc.pl
}

it is important to formulate a dynamical cosmological term without violating the covariance of the action. For example, models with a slowly rolling homogeneous cosmological scalar field provide a popular alternative to the standard time-independent cosmological constant. We can study the simultaneous evolution of the background expansion and an evolution of the scalar field with the self-interacting potential [4].

In this paper we are going to push forward the idea of the emergent running cosmological term from a covariant theory [5]. Parametrization of the cosmological term is derived directly from a formulation of the model in the Einstein frame by means of the Palatini variational approach. In analogy with Starobinsky's purely metric formulation [6], we obtain the parametrization of the cosmological term directly from the potential of the scalar field which appears after formulation of the specific FRW model in the Einstein frame. As a next step we investigate the dynamics of the model with such a form of the dark energy.

In this letter, we demonstrate how $f(R)$ model is modified in the Palatini formulation. Our construction provides a simple model of an evolving dark energy (running cosmological term) to explain a dynamical relaxation of the vacuum energy (gravitational repulsive pressure) to a very small value today (cosmological constant problem [7]). This model, when studied in the Einstein frame, leads also to a small deviation from the $w=-1$ prediction of the non-running dark energy.

\section{Cosmological equations for the polynomial $f(R)$ theory in the Palatini formalism}

The Palatini gravity action for $f(\hat{R})$ gravity is given by

$$
S=S_{\mathrm{g}}+S_{\mathrm{m}}=\frac{1}{2} \int \sqrt{-g} f(\hat{R}) \mathrm{d}^{4} x+S_{\mathrm{m}}
$$


where $\hat{R}$ is the generalized Ricci scalar [8,9]. From the action (1) we get

$f^{\prime}(\hat{R}) \hat{R}_{(\mu \nu)}-\frac{1}{2} f(\hat{R}) g_{\mu \nu}=T_{\mu \nu}$,

$\hat{\nabla}_{\alpha}\left(\sqrt{-g} f^{\prime}(\hat{R}) g^{\mu \nu}\right)=0$,

where $T_{\mu \nu}$ is energy-momentum tensor and $\hat{\nabla}_{\alpha}$ is the covariant derivative calculated with respect to $\Gamma$.

If we take the trace of Eq. (2), we get a structural equation, which is given by

$f^{\prime}(\hat{R}) \hat{R}-2 f(\hat{R})=T$,

where $T=g^{\mu \nu} T_{\mu \nu}$. We assume the FRW metric in the following form:

$\mathrm{d} s^{2}=\mathrm{d} t^{2}-a^{2}(t)\left[\frac{1}{1-k r^{2}} \mathrm{~d} r^{2}+r^{2}\left(\mathrm{~d} \theta^{2}+\sin ^{2} \theta \mathrm{d} \phi^{2}\right)\right]$,

where $a(t)$ is the scale factor, $k$ is a constant of spatial curvature $(k=0, \pm 1)$ and $t$ is the cosmological time. Thereafter, we assume the flat model $(k=0)$.

We assume the energy-momentum tensor for a perfect fluid,

$T_{v}^{\mu}=\operatorname{diag}(-\rho, p, p, p)$,

where $p=w \rho$ with $w=$ const. The conservation condition $T_{v ; \mu}^{\mu}=0[10]$ gives

$\dot{\rho}_{\mathrm{m}}=-3(1+w) H \rho_{\mathrm{m}}$

where $H$ is the Hubble function and $\rho_{\mathrm{m}}$ is the density of baryonic and dark matter which is assumed to be in the form of dust $(w=0)$.

In our paper the function $f(\hat{R})$ is assumed in the polynomial form as

$f(\hat{R})=\sum_{i=1}^{n} \gamma_{i} \hat{R}^{i}$,

where $\gamma_{i}$ are some dimensionful parameters.

Therefore, we introduce more convenient dimensionless functions and parameters,

$$
\begin{aligned}
\Omega_{\mathrm{R}} & =\frac{\hat{R}}{3 H_{0}^{2}}, \quad \Omega_{\gamma_{i}}=3^{i-1} \gamma_{i} H_{0}^{2(i-1)}, \\
\Omega_{\mathrm{tot}} & =\Omega_{\mathrm{m}, 0} a^{-3}+\Omega_{\Lambda, 0}, \quad b=f^{\prime}(\hat{R})=\sum_{i=1}^{n} i \Omega_{\gamma_{i}} \Omega_{R}^{i-1}, \\
d= & -3\left(\sum_{i=1}^{n}(i-2) \Omega_{\gamma_{i}} \Omega_{R}^{i-1}+\frac{4 \Omega_{\Lambda}}{\Omega_{R}}\right) \\
& \times \frac{\sum_{i=1}^{n} i(i-1) \Omega_{\gamma_{i}} \Omega_{R}^{i-1}}{\sum_{i=1}^{n} i(i-2) \Omega_{\gamma_{i}} \Omega_{R}^{i-1}},
\end{aligned}
$$

where $H_{0}$ is the present value of the Hubble function, $\Omega_{\mathrm{m}, 0}=$ $\frac{\rho_{\mathrm{m}, 0}}{3 H_{0}^{2}}, \Omega_{\Lambda, 0}=\frac{\rho_{\Lambda, 0}}{3 H_{0}^{2}} \cdot 1$

For the function (8) the structural equation (4) is in the following form:

$\sum_{i=1}^{n}(i-2) \Omega_{\gamma_{i}} \Omega_{R}^{i}=-\Omega_{\mathrm{m}}-4 \Omega_{\Lambda}$.

The Friedmann equation for the function (8) has the following form:

$$
\begin{array}{r}
\frac{H^{2}}{H_{0}^{2}}=\frac{b^{2}}{\left(b+\frac{d}{2}\right)^{2}}\left[\frac { 1 } { 2 b } \left[\sum_{i=1}^{n} \Omega_{\gamma_{i}} \Omega_{R}^{i-1}\left(\Omega_{R}-2 i \Omega_{\mathrm{tot}}\right)\right.\right. \\
\left.\left.+\Omega_{\mathrm{tot}}-3 \Omega_{\Lambda}\right]+\Omega_{\mathrm{tot}}\right] .
\end{array}
$$

\section{Singularities in the polynomial $f(R)$ theory in the Palatini formalism}

The Friedmann equation (11) can be rewritten in the equivalent form

$a^{\prime 2}=-2 V(a)$,

where $^{\prime}=\frac{\mathrm{d}}{\mathrm{d} \tau}=\frac{|b+d / 2|}{|b|} \frac{\mathrm{d}}{\mathrm{d} t}$ is a new parametrization of time (this parametrization is not a diffeomorphism) and

$$
\begin{array}{r}
V(a)=-\frac{H_{0}^{2} a^{2}}{2}\left[\frac { 1 } { 2 b } \left[\sum_{i=1}^{n} \Omega_{\gamma_{i}} \Omega_{R}^{i-1}\left(\Omega_{R}-2 i \Omega_{\mathrm{tot}}\right)\right.\right. \\
\left.\left.+\Omega_{\mathrm{tot}}-3 \Omega_{\Lambda}\right]+\Omega_{\mathrm{tot}}\right] .
\end{array}
$$

The potential $V(a)$ can be used to construction of a phase space portrait. In this case the phase space is twodimensional,

$\left\{\left(a, a^{\prime}\right): \frac{a^{\prime 2}}{2}+V(a)=-\frac{k}{2}\right\}$.

\footnotetext{
${ }^{1}$ For the sake of generality (following the standard cosmological model) the presence of the cosmological constant is also assumed.
} 


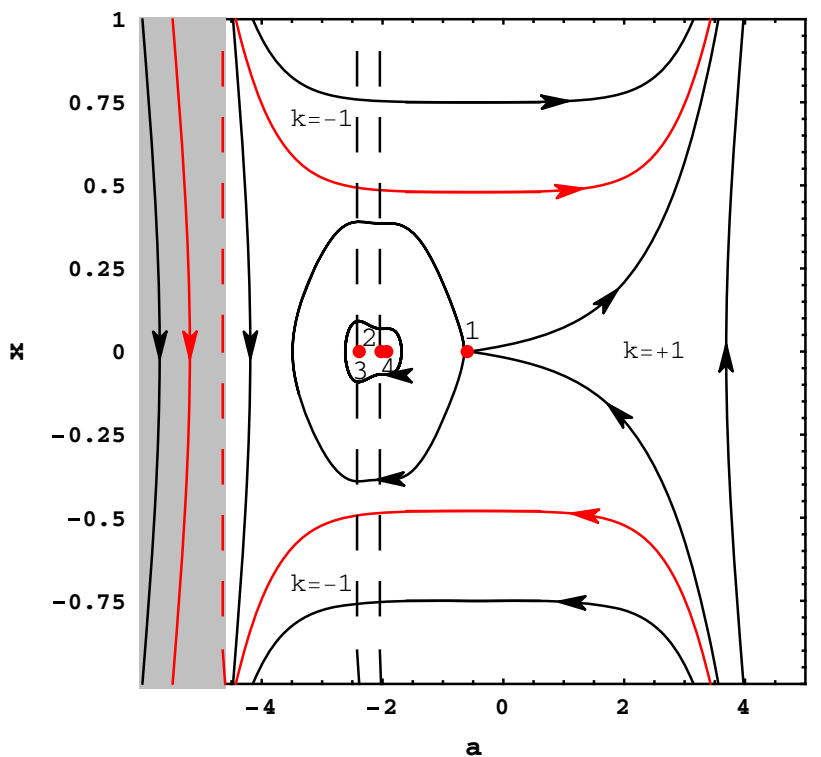

Fig. 1 The phase portrait for system (15)-(16) with $f(\hat{R})=\hat{R}+$ $\gamma \hat{R}^{2}+\delta \hat{R}^{3}$, where $\gamma=10^{-6}\left(\frac{\mathrm{s}^{2} \mathrm{Mpc}^{2}}{\mathrm{~km}^{2}}\right)$ and $\delta=-10^{-14}\left(\frac{\mathrm{s}^{4} \mathrm{Mpc}^{4}}{\mathrm{~km}^{4}}\right)$. Critical points (1), (2), (3) and (4) represent the static Einstein universes. Critical points (1) and (2) are of saddle type and critical points (3) and (4) are of center type. The red dashed line presents the sudden singularity. The black dashed lines present the freeze singularities. The gray color marks the non-physical domain $\left(f^{\prime}(R)<0\right)$. The red trajectories represent the path of evolution for the flat universe. These trajectories separate the domain with the negative curvature $(k=-1)$ from the domain with the positive curvature $(k=+1)$. The scale factor is expressed in a logarithmic scale

The dynamical system has the following form:

$a^{\prime}=x$,

$x^{\prime}=-\frac{\partial V(a)}{\partial a}$.

We assume that the potential function, except some isolated (singular) points, belongs to the class $C^{2}\left(\mathbb{R}_{+}\right)$.

The example phase portraits for the dynamical system (15)-(16) are presented in Figs. 1, 2 and 3.

The evolution of a universe can be treated as a motion of a fictitious particle of unit mass in the potential $V(a)$. Here $a(t)$ plays the role of a positional variable. Equation of motion (16) assumes the form analogous to the Newtonian equation of motion. In this case the lines $\frac{x^{2}}{2}+V(a)=-\frac{k}{2}$ represent possible evolutions of the universe for different initial conditions.

In our model, there are two types of singularities: the freeze and sudden singularities. They are a consequence of the Palatini formalism. We get the freeze singularity when $b+d / 2=0$. The sudden singularity appears when $b=0$ or $b+d / 2$ is equal to infinity.

For the case when the positive part of $f(\hat{R})$ dominates after the domination of the negative part of $f(\hat{R})$, it is possible that

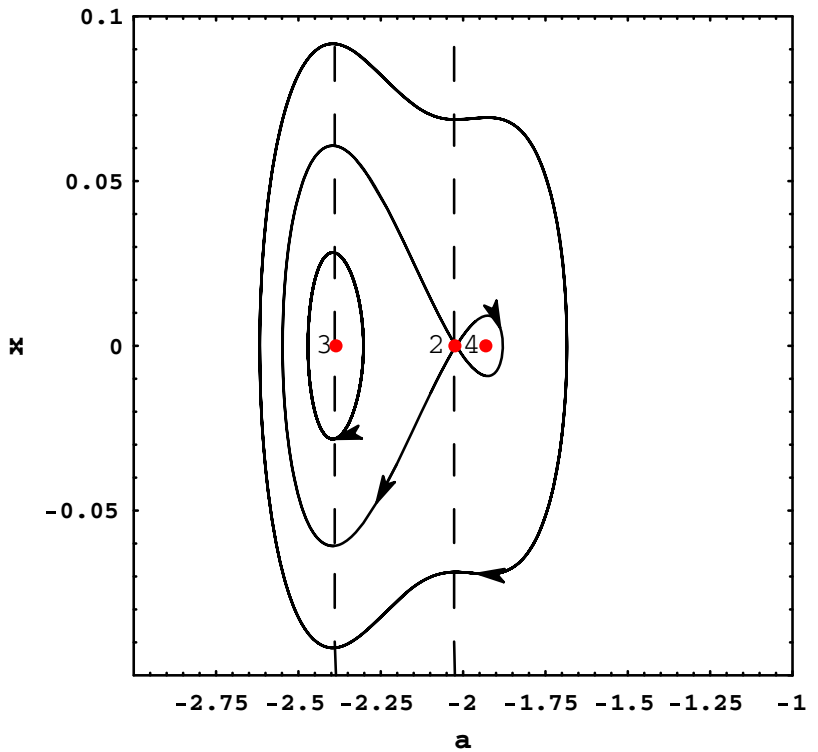

Fig. 2 The zoomed region of Fig. 1. The behavior of trajectories in the neighborhood of critical points (2), (3) and (4) which represent the static Einstein universes. Critical point (2) is of saddle type and critical points (3) and (4) are of center type. The black dashed lines present the freeze singularities. The scale factor is expressed in a logarithmic scale. The homoclinic orbits represent the bouncing models, which evolution starts and ends at the Einstein universe (critical point 2). In the domain bounded by the homoclinic orbits the oscillating models present cases without the initial singularity

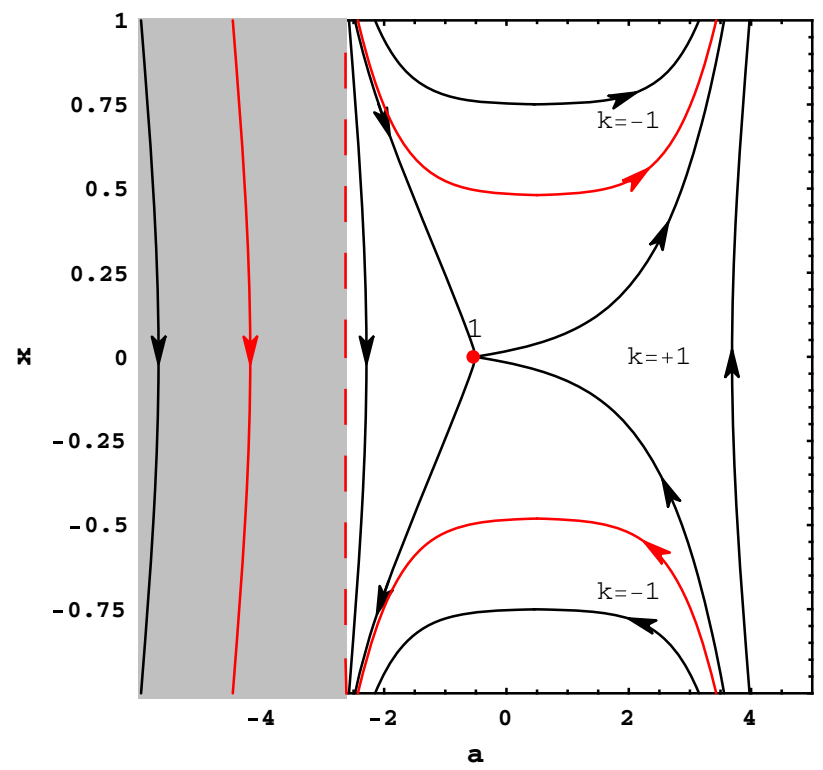

Fig. 3 The phase portrait for system (15)-(16) with $f(\hat{R})=\hat{R}+$ $\gamma \hat{R}+\delta \hat{R}^{3}$, where $\gamma=-10^{-6}\left(\frac{\mathrm{s}^{2} \mathrm{Mpc}^{2}}{\mathrm{~km}^{2}}\right)$ and $\delta=-10^{-14}\left(\frac{\mathrm{s}^{4} \mathrm{Mpc}^{4}}{\mathrm{~km}^{4}}\right)$. Critical point (1), which is of saddle type, represents the static Einstein universe. The red dashed line presents the sudden singularity. The gray color presents the non-physical domain $\left(f^{\prime}(R)<0\right)$. The red trajectories represent the path of evolution for the flat universe. These trajectories separate the domain with the negative curvature $(k=-1)$ from the domain with the positive curvature $(k=+1)$. The scale factor is expressed in a logarithmic scale 


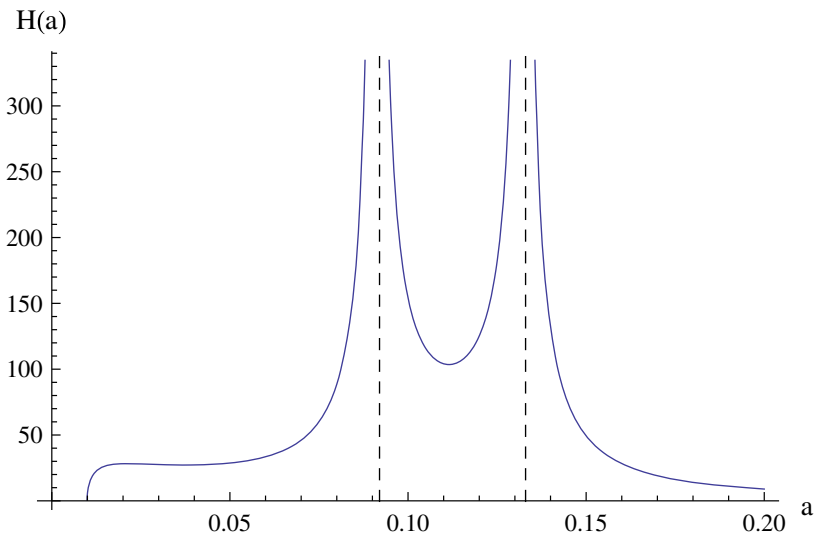

Fig. 4 The evolution of the Hubble function for $f(\hat{R})=\hat{R}+\gamma \hat{R}^{2}+$ $\delta \hat{R}^{3}$, where $\gamma=10^{-6}\left(\frac{\mathrm{s}^{2} \mathrm{Mpc}^{2}}{\mathrm{~km}^{2}}\right)$ and $\delta=-10^{-14}\left(\frac{\mathrm{s}^{4} \mathrm{Mpc}^{4}}{\mathrm{~km}^{4}}\right)$. The black dashed lines present the freeze singularities. Note that the singularity of the big bang type does not appear here. $H(a)$ is expressed in units of $\frac{100 \mathrm{~km}}{\mathrm{~s} \mathrm{Mpc}}$

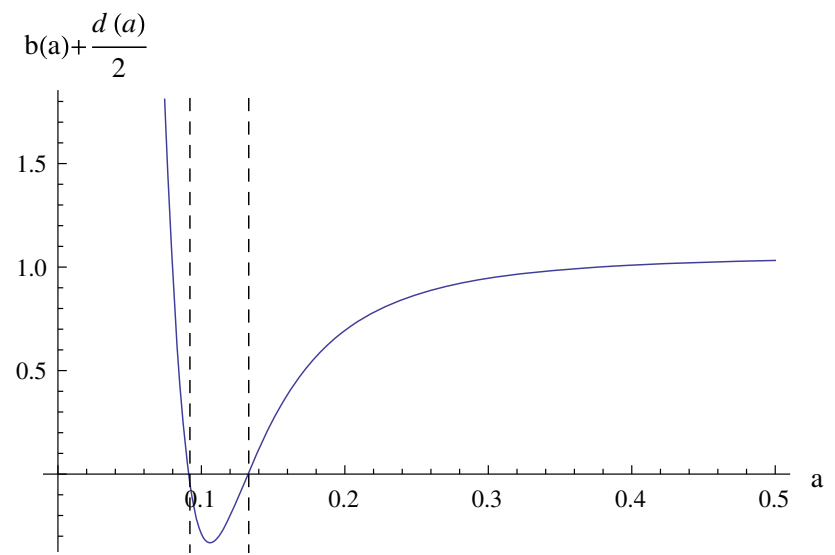

Fig. 5 The evolution of $b(a)+\frac{d(a)}{2}$. For values of the scale factor for which the equation $b(a)+\frac{d(a)}{2}=0$ has roots, the freeze singularities appear ( black dashed lines). This figure corresponds with Fig. 4

two freeze singularities appear. This situation is presented in Fig. 4 for $f(\hat{R})=\hat{R}+10^{-2} \hat{R}^{2}-10^{-6} \hat{R}^{3}$. In this case they appear two freeze singularities and one sudden singularity. The evolution of $b(a)+\frac{d(a)}{2}$, which corresponds with Fig. 4 , is presented in Fig. 5. Note that, for values of the scale factor for which the function $b(a)+\frac{d(a)}{2}$ has roots, the freeze singularities appear. $V(a)$ potential, which corresponds with Fig. 4, is presented in Figs. 6 and 7.

\section{Singularities in the Palatini $f(R)=R+\gamma R^{2}+\delta R^{3}$ model}

For the special case of polynomial $f(\hat{R})=\hat{R}+\gamma \hat{R}^{2}+\delta \hat{R}^{3}$, one gets the following structural equation:

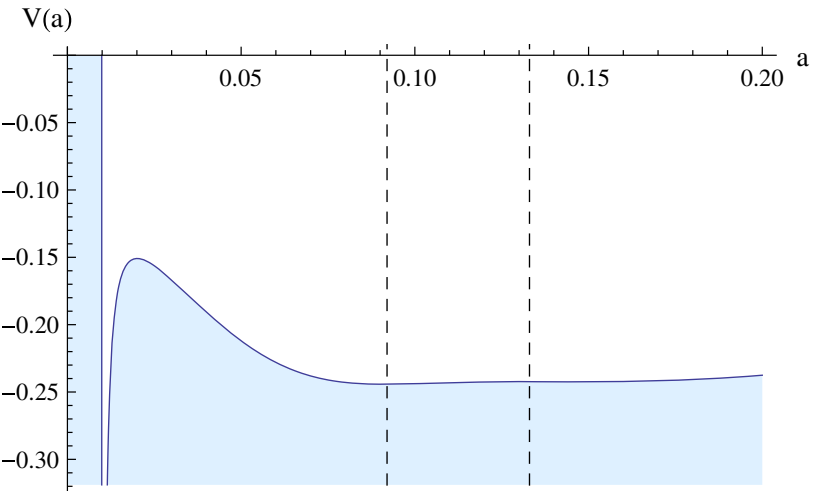

Fig. 6 The evolution of $V(a)$. This figure corresponds with Fig. 4. The black dashed lines present the freeze singularities. The potential is regular at these singularities while its higher derivative blows up. The pole of $V(a)$ represents the sudden singularity. The potential $V(a)$ is expressed in units of $\frac{10^{4} \mathrm{~km}^{2}}{\mathrm{~s}^{2} \mathrm{Mpc}^{2}}$

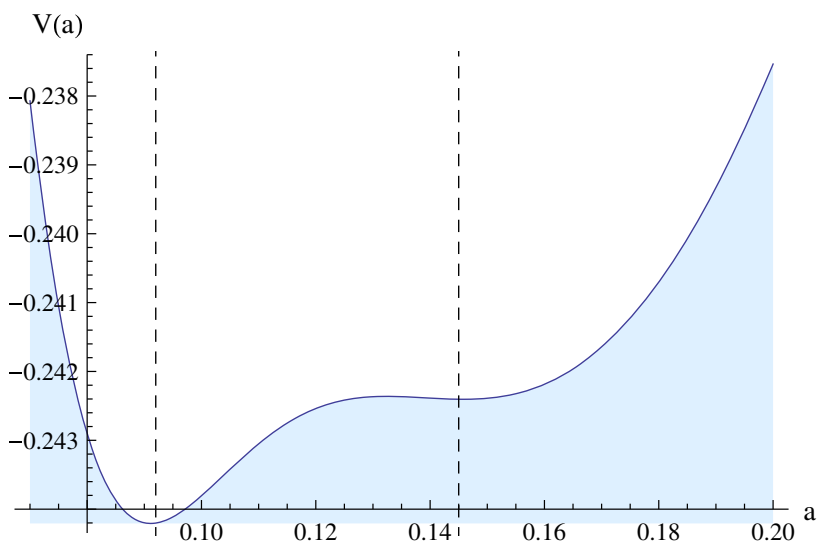

Fig. 7 The zoomed region of Fig. 6. The extrema of $V(a)$ are presented. The black dashed lines represent the freeze singularities. The potential $V(a)$ is expressed in units of $\frac{10^{4} \mathrm{~km}^{2}}{\mathrm{~s}^{2} \mathrm{Mpc}^{2}}$

$\Omega_{R}-\Omega_{\delta} \Omega_{R}^{3}=\Omega_{\mathrm{m}}+4 \Omega_{\Lambda}$,

where $\Omega_{\gamma}=3 \gamma H_{0}^{2}$ and $\Omega_{\delta}=9 \delta H_{0}^{4}$.

The Friedmann equation takes the form

$$
\begin{aligned}
\frac{H^{2}}{H_{0}^{2}}= & \frac{b^{2}}{\left(b+\frac{d}{2}\right)^{2}} \times\left[\frac { \Omega _ { \mathrm { R } } } { 2 b } \left[\Omega_{\gamma}\left(\Omega_{\mathrm{R}}-4 \Omega_{\mathrm{tot}}\right)\right.\right. \\
& \left.\left.\left.+2 \Omega_{\delta} \Omega_{\mathrm{R}}\left(\Omega_{\mathrm{R}}-3 \Omega_{\mathrm{tot}}\right)\right)\right]+\Omega_{\mathrm{tot}}+\Omega_{k}\right],
\end{aligned}
$$

where

$$
\begin{aligned}
& \Omega_{\mathrm{tot}}=\Omega_{m, 0} a^{-3}+\Omega_{\Lambda, 0}, \\
& b=f^{\prime}(\hat{R})=1+\Omega_{\mathrm{R}}\left[2 \Omega_{\gamma}+3 \Omega_{\delta} \Omega_{\mathrm{R}}\right], \\
& d=\frac{1}{H} \frac{\mathrm{d} b}{\mathrm{~d} t}=6 \frac{\Omega_{\gamma}+3 \Omega_{\delta} \Omega_{\mathrm{R}}}{3 \Omega_{\delta} \Omega_{\mathrm{R}}^{2}-1}\left[\Omega_{\mathrm{R}}\left(1-\Omega_{\delta} \Omega_{\mathrm{R}}^{2}\right)-4 \Omega_{\Lambda, 0}\right] .
\end{aligned}
$$


The condition for the appearance of the freeze singularity is $b+\frac{d}{2}=0$ and in this case it has the form

$3 \Omega_{\gamma} \Omega_{\delta} \Omega_{R}^{3}+9 \Omega_{\delta} \Omega_{R}^{2}+\left(\Omega_{\gamma}-36 \Omega_{\delta} \Omega_{\Lambda}\right) \Omega_{R}-12 \Omega_{\gamma} \Omega_{\Lambda}-1=0$.

Equation (20) has the following solution:

$$
\begin{aligned}
\Omega_{\mathrm{R}_{\text {sing }}}= & \Omega_{\gamma}^{-1}\left[-1+\frac{r\left(\Omega_{\gamma}, \Omega_{\delta}, \Omega_{\Lambda}\right)}{92^{1 / 3} \Omega_{\delta}}\right. \\
& \left.-\frac{2^{1 / 3}\left(-81 \Omega_{\delta}^{2}+9 \Omega_{\gamma} \Omega_{\delta}\left(\Omega_{\gamma}-36 \Omega_{\delta} \Omega_{\Lambda}\right)\right)}{9 r\left(\Omega_{\gamma}, \Omega_{\delta}, \Omega_{\Lambda}\right) \Omega_{\delta}}\right],
\end{aligned}
$$

where

$$
\begin{aligned}
r\left(\Omega_{\gamma}, \Omega_{\delta}, \Omega_{\Lambda}\right) \\
=2\left[243 \Omega_{\gamma}^{2} \Omega_{\delta}^{2}\left(1+6 \Omega_{\gamma} \Omega_{\Lambda}\right)-729 \Omega_{\delta}^{3}\left(1+6 \Omega_{\gamma} \Omega_{\Lambda}\right)\right. \\
\quad+\left(59049\left(\Omega_{\gamma}^{2}-3 \Omega_{\delta}\right)^{2} \Omega_{\delta}^{4}\left(1+6 \Omega_{\gamma} \Omega_{\Lambda}\right)^{2}\right. \\
\left.\left.\quad-\left(81 \Omega_{\delta}^{2}-9 \Omega_{\gamma} \Omega_{\delta}\left(\Omega_{\gamma}-36 \Omega_{\delta} \Omega_{\Lambda}\right)\right)^{3}\right)^{1 / 2}\right]^{1 / 3}
\end{aligned}
$$

For the sudden singularity the condition $b=0$ provides the equation

$1+\Omega_{\mathrm{R}}\left[2 \Omega_{\gamma}+3 \Omega_{\delta} \Omega_{\mathrm{R}}\right]=0$.

which has the following solutions:

$\Omega_{\mathrm{R}_{\text {sing }}}=\frac{-\Omega_{\gamma} \pm \sqrt{\Omega_{\gamma}^{2}-3 \Omega_{\delta}}}{3 \Omega_{\delta}}$

\section{The Palatini approach in the Einstein frame}

If $f^{\prime \prime}(\hat{R}) \neq 0$ then the action (1) can be rewritten in dynamically equivalent form of the first order Palatini gravitational action [11-13]

$$
\begin{aligned}
S\left(g_{\mu \nu}, \Gamma_{\rho \sigma}^{\lambda}, \chi\right)= & \frac{1}{2} \int \mathrm{d}^{4} x \sqrt{-g}\left(f^{\prime}(\chi)(\hat{R}-\chi)+f(\chi)\right) \\
& +S_{m}\left(g_{\mu \nu}, \psi\right) .
\end{aligned}
$$

The conditions that allow for the change of variables and lead to Eq. (25) were discussed in the well-known paper of Olmo [14], who clarified the issues raised by Faraoni [15].

Let $\Phi=f^{\prime}(\chi)$ be a scalar field, where $\chi=\hat{R}$. Then the action (25) takes the form

$$
\begin{aligned}
S\left(g_{\mu \nu}, \Gamma_{\rho \sigma}^{\lambda}, \Phi\right)= & \frac{1}{2} \int \mathrm{d}^{4} x \sqrt{-g}(\Phi \hat{R}-U(\Phi)) \\
& +S_{m}\left(g_{\mu \nu}, \psi\right),
\end{aligned}
$$

where the potential $U(\Phi)$ is given as

$U_{f}(\Phi) \equiv U(\Phi)=\chi(\Phi) \Phi-f(\chi(\Phi))$

with $\Phi=\frac{\mathrm{d} f(\chi)}{\mathrm{d} \chi}$ and $\hat{R} \equiv \chi=\frac{\mathrm{d} U(\Phi)}{\mathrm{d} \Phi}$.
After the Palatini variation of the action (26) we get the following equations of motion:

$$
\begin{aligned}
& \Phi\left(\hat{R}_{\mu \nu}-\frac{1}{2} g_{\mu \nu} \hat{R}\right)+\frac{1}{2} g_{\mu \nu} U(\Phi)-T_{\mu \nu}=0, \\
& \hat{\nabla}_{\lambda}\left(\sqrt{-g} \Phi g^{\mu \nu}\right)=0 \\
& \hat{R}-U^{\prime}(\Phi)=0 .
\end{aligned}
$$

As a consequence of (28b) the connection $\hat{\Gamma}$ is a metric connection for a new (conformally related) metric $\bar{g}_{\mu \nu}=\Phi g_{\mu \nu}$; thus $\hat{R}_{\mu \nu}=\bar{R}_{\mu \nu}, \bar{R}=\bar{g}^{\mu \nu} \bar{R}_{\mu \nu}=\Phi^{-1} \hat{R}$ and $\bar{g}_{\mu \nu} \bar{R}=$ $g_{\mu \nu} \hat{R}$. The $g$-trace of (28a) gives a new structural equation

$2 U(\Phi)-U^{\prime}(\Phi) \Phi=T$

Equations (28a) and (28c) can be rewritten in the following form:

$\bar{R}_{\mu \nu}-\frac{1}{2} \bar{g}_{\mu \nu} \bar{R}=\bar{T}_{\mu \nu}-\frac{1}{2} \bar{g}_{\mu \nu} \bar{U}(\Phi)$,

$\Phi \bar{R}-\left(\Phi^{2} \bar{U}(\Phi)\right)^{\prime}=0$

where $\bar{U}(\phi)=U(\phi) / \Phi^{2}, \bar{T}_{\mu \nu}=\Phi^{-1} T_{\mu \nu}$. In this case, the structural equation is given by the following formula:

$\Phi \bar{U}^{\prime}(\Phi)+\bar{T}=0$

The action for the metric $\bar{g}_{\mu \nu}$ and the scalar field $\Phi$ can be recast into the Einstein frame form

$S\left(\bar{g}_{\mu \nu}, \Phi\right)=\frac{1}{2} \int \mathrm{d}^{4} x \sqrt{-\bar{g}}(\bar{R}-\bar{U}(\Phi))+S_{m}\left(\Phi^{-1} \bar{g}_{\mu \nu}, \psi\right)$

with non-minimal coupling between $\Phi$ and $\bar{g}_{\mu \nu}$

$\bar{T}^{\mu \nu}=-\frac{2}{\sqrt{-\bar{g}}} \frac{\delta}{\delta \bar{g}_{\mu \nu}} S_{m}=(\bar{\rho}+\bar{p}) \bar{u}^{\mu} \bar{u}^{\nu}+\bar{p} \bar{g}^{\mu \nu}=\Phi^{-3} T^{\mu \nu}$,

$\bar{u}^{\mu}=\Phi^{-\frac{1}{2}} u^{\mu}, \bar{\rho}=\Phi^{-2} \rho, \bar{p}=\Phi^{-2} p, \bar{T}_{\mu \nu}=$ $\Phi^{-1} T_{\mu \nu}, \bar{T}=\Phi^{-2} T$ (see e.g. $[13,16]$ ).

The metric $\bar{g}_{\mu \nu}$ takes the standard FRW form

$\mathrm{d} \bar{s}^{2}=-\mathrm{d} \bar{t}^{2}+\bar{a}^{2}(\bar{t})\left[\mathrm{d} r^{2}+r^{2}\left(\mathrm{~d} \theta^{2}+\sin ^{2} \theta \mathrm{d} \phi^{2}\right)\right]$,

where $\mathrm{d} \bar{t}=\Phi(t)^{\frac{1}{2}} \mathrm{~d} t$ and a new scale factor $\bar{a}(\bar{t})=$ $\Phi(\bar{t})^{\frac{1}{2}} a(\bar{t})$. In the case of barotropic matter, the cosmological equations are

$3 \bar{H}^{2}=\bar{\rho}_{\Phi}+\bar{\rho}_{\mathrm{m}}, \quad 6 \frac{\ddot{a}}{\bar{a}}=2 \bar{\rho}_{\Phi}-\bar{\rho}_{\mathrm{m}}(1+3 w)$ 
where

$\bar{\rho}_{\Phi}=\frac{1}{2} \bar{U}(\Phi), \quad \bar{\rho}_{\mathrm{m}}=\rho_{0} \bar{a}^{-3(1+w)} \Phi^{\frac{1}{2}(3 w-1)}$

and $w=\bar{p}_{\mathrm{m}} / \bar{\rho}_{\mathrm{m}}=p_{\mathrm{m}} / \rho_{\mathrm{m}}$. In this case, the conservation equation has the following form:

$\dot{\bar{\rho}}_{\mathrm{m}}+3 \bar{H} \bar{\rho}_{\mathrm{m}}(1+w)=-\dot{\bar{\rho}}_{\Phi}$

Let us consider our Palatini model $f(\hat{R})=\sum_{i=1}^{n} \gamma_{i} \hat{R}^{i}$ in the Einstein frame, where $\gamma_{1}=1$. The potential $\bar{U}$ is given by the following formula:

$\bar{U}(\hat{R})=2 \bar{\rho}_{\Phi}(\hat{R})=\frac{\sum_{i=1}^{n}(i-1) \gamma_{i} \hat{R}^{i}}{\left(\sum_{i=1}^{n} i \gamma_{i} \hat{R}^{i-1}\right)^{2}}$.

The scalar field $\Phi$ can be parametrized by $\hat{R}$ in the following way:

$\Phi(\hat{R})=\frac{\mathrm{d} f(\hat{R})}{\mathrm{d} \hat{R}}=\sum_{i=1}^{n} i \gamma_{i} \hat{R}^{i-1}$.

The relation between $\bar{U}$ and $\hat{R}$ for the case $f(\hat{R})=\hat{R}+$ $\gamma \hat{R}^{2}+\delta \hat{R}^{3}$ is presented in Fig. 8 .

In this frame, two scenarios of cosmic evolution may appear. In the first one the evolution of the universe starts from the generalized sudden singularity. The second case is when it starts from the freeze singularity. The diagrams of the corresponding Newtonian potentials $V(\bar{a})$ are presented in Figs. 9 and 10. We can use the potential $V(\bar{a})$ to construct phase space portraits analogous to the ones in Sect. 3 (see Figs. 11, 12).

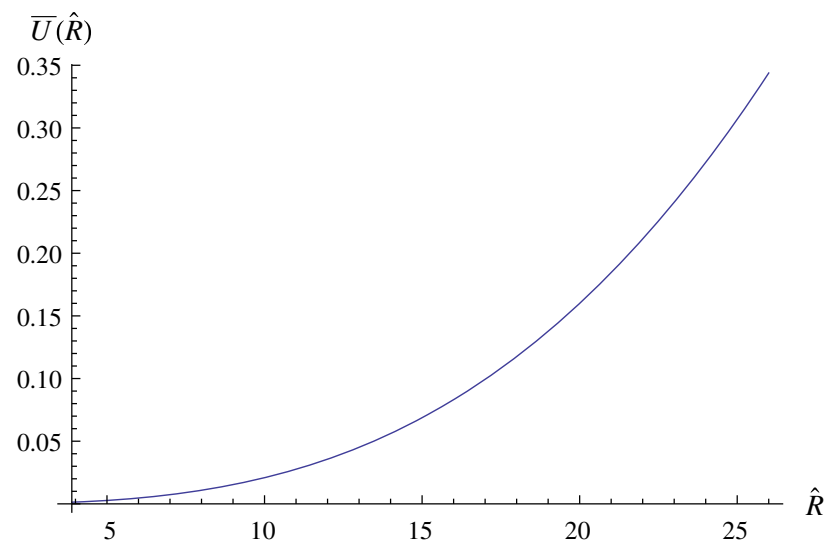

Fig. 8 The evolution of $\bar{U}(\hat{R})$ in the Einstein frame in the case when the evolution of the universe starts from the generalized sudden singularity. For illustration it is assumed that $f(\hat{R})=\hat{R}+\gamma \hat{R}^{2}+\delta \hat{R}^{3}$, where $\gamma=10^{-9}\left(\frac{\mathrm{s}^{2} \mathrm{Mpc}^{2}}{\mathrm{~km}^{2}}\right)$ and $\delta=10^{-13}\left(\frac{\mathrm{s}^{4} \mathrm{Mpc}^{4}}{\mathrm{~km}^{4}}\right) \cdot \bar{U}(\hat{R})$ is expressed in units of $\frac{10^{4} \mathrm{~km}^{2}}{\mathrm{~s}^{2} \mathrm{Mpc}^{2}}$
$\mathrm{V}(\bar{a})$

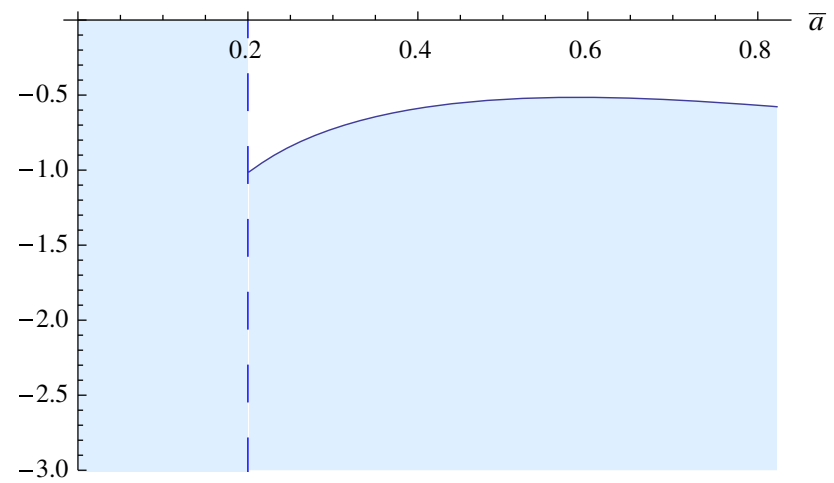

Fig. 9 The evolution of $V(\bar{a})$ in the Einstein frame in the case when the evolution of the universe starts from the generalized sudden singularity. For illustration it is assumed that $f(\hat{R})=\hat{R}+\gamma \hat{R}^{2}+\delta \hat{R}^{3}$. This figure corresponds to Fig. 11. The blue dashed line presents the generalized sudden singularity. Note that the undesirable freeze singularity disappears. The potential $V(a)$ is expressed in units of $\frac{10^{4} \mathrm{~km}^{2}}{\mathrm{~s}^{2} \mathrm{Mpc}^{2}}$

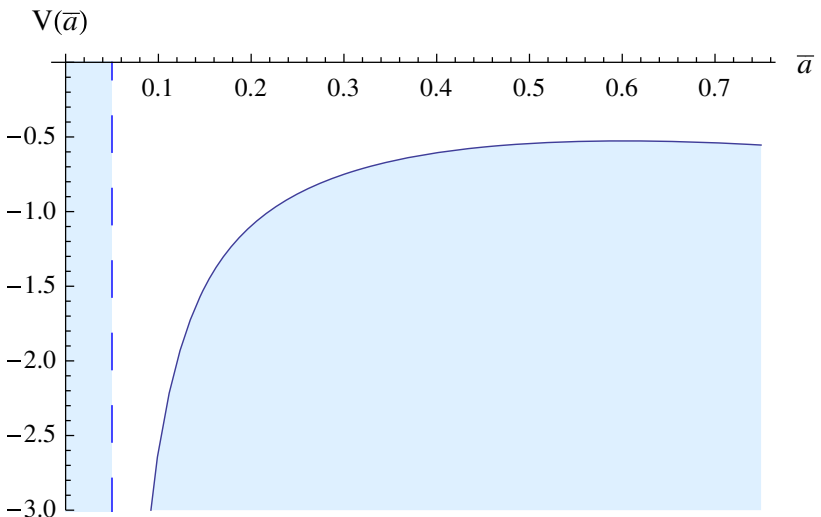

Fig. 10 The evolution of $V(\bar{a})$ in the Einstein frame in the case when the evolution of the universe starts from the freeze singularity. For illustration it is assumed that $f(\hat{R})=\hat{R}+\gamma \hat{R}^{2}+\delta \hat{R}^{3}$. This figure corresponds with Fig. 12. The blue dashed line presents the freeze singularity. The potential $V(a)$ is expressed in units of $\frac{10^{4} \mathrm{~km}^{2}}{\mathrm{~s}^{2} \mathrm{Mpc}^{2}}$

The evolution of the scalar field potential $\bar{U}(\bar{t})$, which plays a role of dynamical cosmological constant, is presented in Fig. 13 for the case with the generalized sudden singularity. Note that for the late time the potential $\bar{U}(\bar{t})$ is constant. The evolution of $\bar{U}(\bar{t})$, for the case when the freeze singularity appears, is presented in Fig. 14. For the late time the potential $\bar{U}(\bar{a})$ can be approximated by

$\bar{U}(\bar{a})=\frac{\gamma \hat{R}(\bar{a})^{2}}{1+4 \gamma \hat{R}(\bar{a})}=\frac{\gamma\left(4 \Lambda+\bar{\rho}_{\mathrm{m}, 0} \bar{a}^{-3}\right)^{2}}{1+4 \gamma\left(4 \Lambda+\bar{\rho}_{\mathrm{m}, 0} \bar{a}^{-3}\right)}$.

From the structural equation (32) for $f(\hat{R})=\hat{R}+\gamma \hat{R}^{2}+$ $\delta \hat{R}^{3}$ case, we get the parameterization of the dust matter density with respect to $\hat{R}$, 


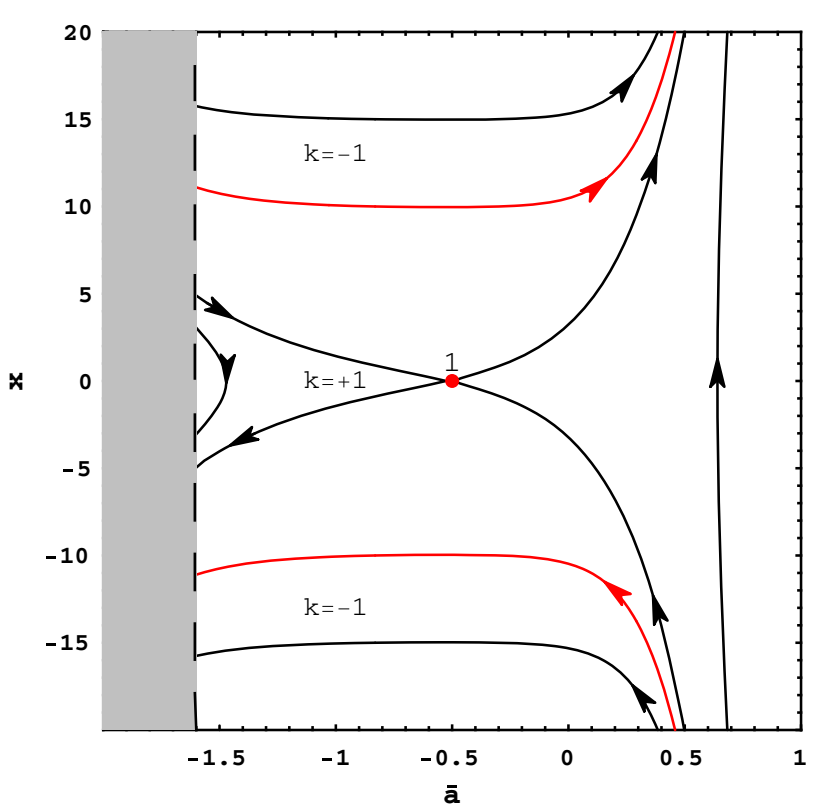

Fig. 11 The phase portrait for system (15)-(16) in the Einstein frame in the case when the evolution of the universe starts from the generalized sudden singularity. For illustration it is assumed that $f(\hat{R})=\hat{R}+\gamma \hat{R}^{2}+$ $\delta \hat{R}^{3}$, where $\gamma=10^{-9}\left(\frac{\mathrm{s}^{2} \mathrm{Mpc}^{2}}{\mathrm{~km}^{2}}\right)$ and $\delta=10^{-13}\left(\frac{\mathrm{s}^{4} \mathrm{Mpc}^{4}}{\mathrm{~km}^{4}}\right)$. Critical point (1) represents the static Einstein universe and is a saddle. The black dashed line presents the generalized sudden singularity. The gray color presents the non-physical domain $\left(\bar{a}<\bar{a}_{\mathrm{S}}\right)$. The red trajectories represent the path of evolution for the flat universe. These trajectories separate the domain with negative curvature $(k=-1)$ from the domain with positive curvature $(k=+1)$. The scale factor is expressed in a logarithmic scale

$\bar{\rho}_{\mathrm{m}}=\frac{\hat{R}-\delta \hat{R}^{3}}{\left(1+2 \gamma \hat{R}+3 \delta \hat{R}^{2}\right)^{2}}-4 \Lambda$.

It is interesting that in the Einstein frame the interaction between dark matter and dark energy naturally appears as a physical phenomenon. This interaction modifies the original scaling law for dust matter by a function $\epsilon(\bar{t})$. We have

$\bar{\rho}_{\mathrm{m}}=\bar{\rho}_{\mathrm{m}, 0} \bar{a}(\bar{t})^{-3+\epsilon(\bar{t})}$,

where $\epsilon=\frac{1}{\ln \bar{a}} \int \frac{Q}{\bar{H} \bar{\rho}_{\mathrm{m}}} d \ln \bar{a}$ and $Q=-\dot{\bar{\rho}}_{\phi}=\bar{H}(\hat{R}) \times$ $\times \bar{\rho}_{\mathrm{m}}(\hat{R}) \frac{\left.3 \hat{R}(\gamma+3 \delta \hat{R})\left(\delta \hat{R}^{2}-1\right)\right)}{\hat{R}(\gamma+3 \delta \hat{R}(3+\gamma \hat{R}))-1}$ for the case $f(\hat{R})=\hat{R}+\gamma \hat{R}^{2}+$ $\delta \hat{R}^{3}$. The evolution of $\epsilon(\bar{t})$ is presented in Fig. 15 .

\section{Conclusions}

The main goal of the paper was to point out some advantages of the formulation of Palatini FRW cosmology in the Einstein frame. The most crucial one is that in the Einstein frame the

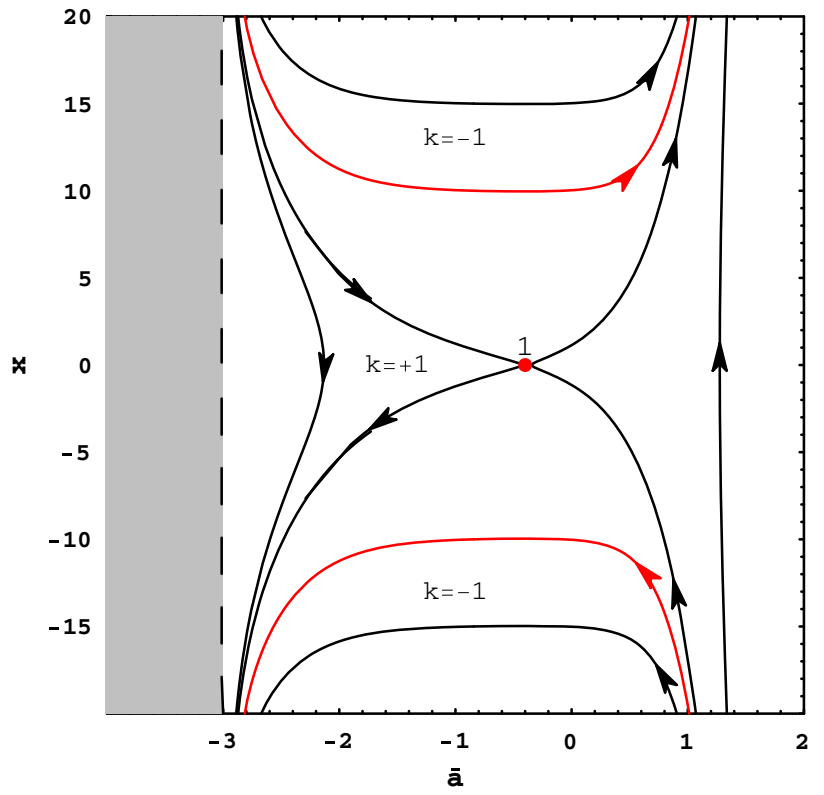

Fig. 12 The phase portrait for system (15)-(16) in the Einstein frame in the case when the evolution of the universe starts from the freeze singularity. For illustration it is assumed that $f(\hat{R})=\hat{R}+\gamma \hat{R}^{2}+\delta \hat{R}^{3}$, where $\gamma=-10^{-9}\left(\frac{\mathrm{s}^{2} \mathrm{Mpc}^{2}}{\mathrm{~km}^{2}}\right)$ and $\delta=-10^{-13}\left(\frac{\mathrm{s}^{4} \mathrm{Mpc}^{4}}{\mathrm{~km}^{4}}\right)$. Critical point (1) represents the static Einstein universe and is a saddle. The black dashed line presents the freeze singularity. The gray color presents the non-physical domain $\left(\bar{a}<\bar{a}_{\mathrm{s}}\right)$. The red trajectories represent the path of evolution for the flat universe. These trajectories separate the domain with negative curvature $(k=-1)$ from the domain with positive curvature $(k=+1)$. The scale factor is expressed in a logarithmic scale

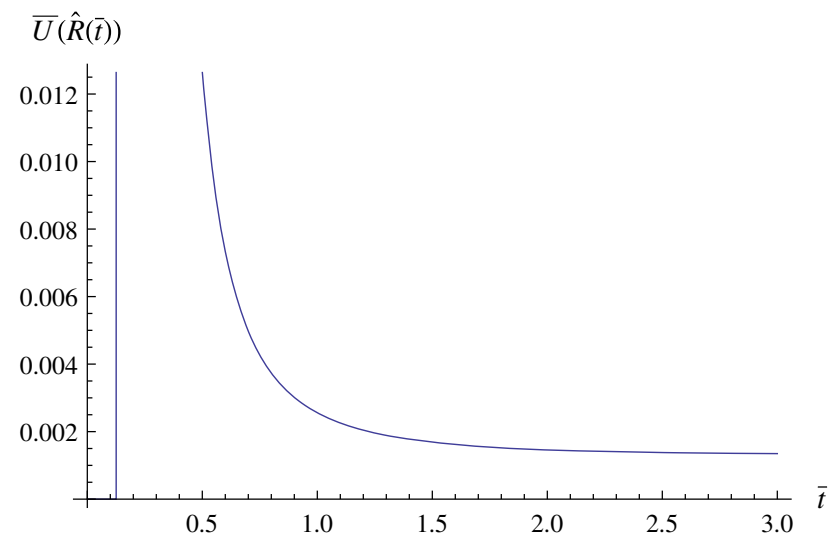

Fig. 13 The evolution of $\bar{U}(\hat{R}(\bar{t}))$ in the Einstein frame in the case when the evolution of the universe starts from the generalized sudden singularity. For illustration it is assumed that $f(\hat{R})=\hat{R}+\gamma \hat{R}^{2}+\delta \hat{R}^{3}$, where $\gamma=10^{-9}\left(\frac{\mathrm{s}^{2} \mathrm{Mpc}^{2}}{\mathrm{~km}^{2}}\right)$ and $\delta=10^{-13}\left(\frac{\mathrm{s}^{4} \mathrm{Mpc}^{4}}{\mathrm{~km}^{4}}\right)$. Note that for the late time the potential $\bar{U}(\bar{t})$ goes to a constant value at late time. Time is expressed in unts of $\frac{\mathrm{s} \mathrm{Mpc}}{100 \mathrm{~km}}$ and $\bar{U}(\hat{R}(\bar{t}))$ is expressed in units of $\frac{10^{4} \mathrm{~km}^{2}}{\mathrm{~s}^{2} \mathrm{Mpc}^{2}}$

parametrization of dark energy is uniquely determined. In general it is obtained in the covariant form as a function of the Ricci scalar. 


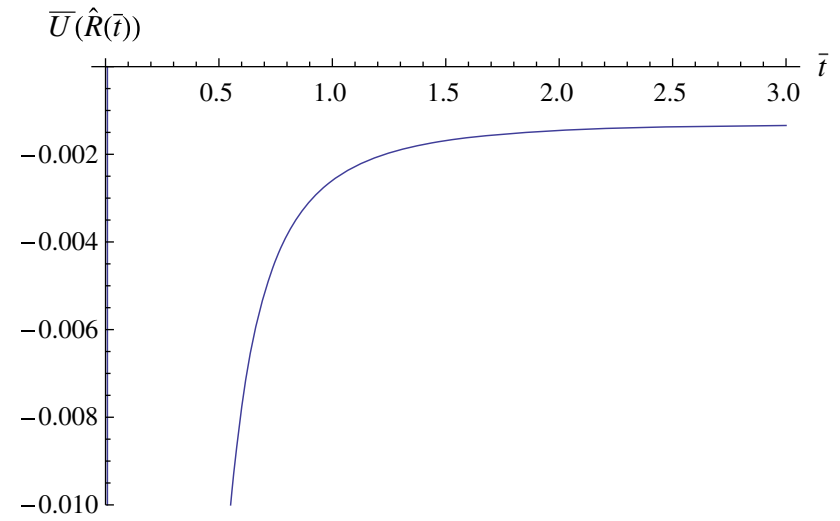

Fig. 14 The evolution of $\bar{U}(\hat{R}(\bar{t}))$ in the Einstein frame in the case when the evolution of the universe starts from the freeze singularity. For illustration it is assumed that $f(\hat{R})=\hat{R}+\gamma \hat{R}^{2}+\delta \hat{R}^{3}$, where $\gamma=-10^{-9}\left(\frac{\mathrm{s}^{2} \mathrm{Mpc}^{2}}{\mathrm{~km}^{2}}\right)$ and $\delta=-10^{-13}\left(\frac{\mathrm{s}^{4} \mathrm{Mpc}^{4}}{\mathrm{~km}^{4}}\right)$. Time is expressed in units of $\frac{\mathrm{s} \mathrm{Mpc}}{100 \mathrm{~km}}$ and $\bar{U}(\hat{R}(\bar{t}))$ is expressed in units of $\frac{10^{4} \mathrm{~km}^{2}}{\mathrm{~s}^{2} \mathrm{Mpc}^{2}}$

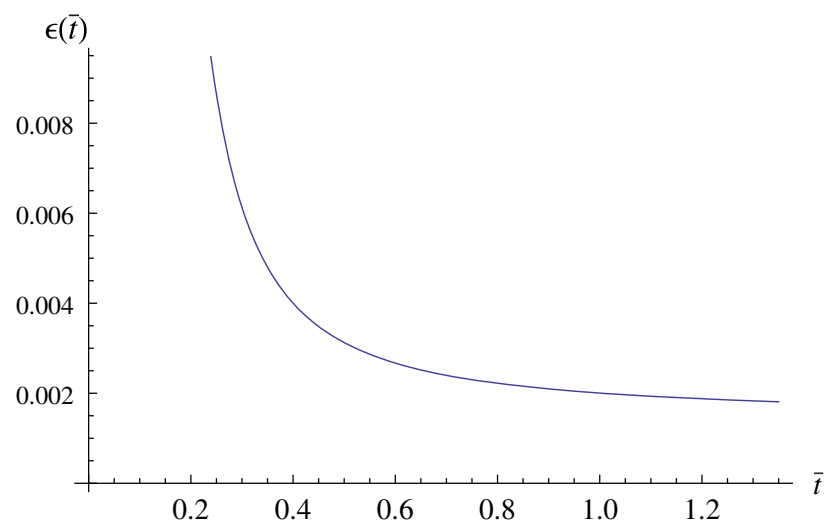

Fig. 15 The evolution of $\epsilon(\bar{t})$ in the Einstein frame in the case when the evolution of the universe starts from the generalized sudden singularity. For illustration it is assumed that $f(\hat{R})=\hat{R}+\gamma \hat{R}^{2}+\delta \hat{R}^{3}$, where $\gamma=10^{-9}\left(\frac{\mathrm{s}^{2} \mathrm{Mpc}^{2}}{\mathrm{~km}^{2}}\right)$ and $\delta=10^{-13}\left(\frac{\mathrm{s}^{4} \mathrm{Mpc}^{4}}{\mathrm{~km}^{4}}\right)$. Note that for the late time $\epsilon(\bar{t})$ is constant. Time is expressed in units of $\frac{\mathrm{s} \mathrm{Mpc}}{100 \mathrm{~km}}$

It is well known that scalar-tensor theories of gravity can be formulated both in the Jordan and in the Einstein frame. These frames are conformally related [17]. We also know that the formulations of a scalar-tensor theory in two different conformal frames, although mathematically equivalent, are physically inequivalent.

In recent years significant progress has been achieved in the understanding of the geometric features of the Palatini theories and the role of the choice of the frame $[18,19]$. In particular, through the analysis of the tensorial perturbations, it is shown that it is the auxiliary (conformal in this case) metric, which determines the propagation of the gravitational waves, while the geodesic motion of the particles is dictated by the Jordan frame metric. A discussion, in this direction, seems to be important as it would help to eliminate the need to choose between the frames.

Faraoni and Gunzing gave a simple argument which favors the Einstein frame over the Jordan frame: in the latter one should potentially detect the time-dependent amplification induced by gravitational waves [20].

An analogous problem has been detected in $f(R)$ gravity: the Jordan frames could be physically non-equivalent, although they are connected by a conformal transformation [21,22]. In principle, there are two types of admissible arguments for favoring one frame over another: coming from observations (for example astronomical observations) or being of a theoretical nature (e.g. showing that some obstacles or pathologies will vanish in the privileged frame).

From our investigation of the model in an Einstein frame we found that some pathologies, like degenerate multiple freeze singularities, [23] disappear in a generic case. The big bang singularity is replaced by the singularity of a finite scale factor. The subtle issue of what a singularity is in the context of Palatini theories has been discussed in recent work by Olmo et al. [24-26]. We are using singularities in a cosmological framework rather as a theoretical discriminator for the optimal choice of the frame. We pointed out that the Einstein frame is favored in this context.

Because the potential $\bar{U}(\hat{R}(\bar{t}))$ is constant for the late time, in the case when matter is negligible, the inflation appears like in the case $f(\hat{R})=\hat{R}+\gamma \hat{R}^{2}$ [23].

There are also some other advantages when transforming to an Einstein frame, namely that in this frame one naturally obtains the formula for dynamical dark energy which is going at late time toward the cosmological constant. It is important that the corresponding parametrization of dark energy is not postulated ad hoc but emerges from first principles - which is the formulation of the problem in the Einstein frame. It is important that the parametrization of dark energy (energy density as well as pressure) in terms of the Ricci scalar is given in a covariant form from the structure equation.

After a transition to the Einstein frame the model evolution is governed by the Friedmann equation with two interacting fluids: dark energy and dark matter. This interaction modifies the standard scaling of the redshift relation for dark matter.

Open Access This article is distributed under the terms of the Creative Commons Attribution 4.0 International License (http://creativecomm ons.org/licenses/by/4.0/), which permits unrestricted use, distribution, and reproduction in any medium, provided you give appropriate credit to the original author(s) and the source, provide a link to the Creative Commons license, and indicate if changes were made.

Funded by SCOAP S $^{3}$

\section{References}

1. J. Martin, C.R. Phys. 13, 566 (2012). doi:10.1016/j.crhy.2012.04. 008 
2. P.J.E. Peebles, B. Ratra, Rev. Mod. Phys. 75, 559 (2003). doi:10. 1103/RevModPhys.75.559

3. A. Stachowski, M. Szydlowski, K. Urbanowski, Eur. Phys. J. C 77(6), 357 (2017). doi:10.1140/epjc/s10052-017-4934-2

4. B. Ratra, P.J.E. Peebles, Phys. Rev. D 37, 3406 (1988). doi:10. 1103/PhysRevD.37.3406

5. A. Stachowski, M. Szydlowski, Eur. Phys. J. C 76(11), 606 (2016). doi:10.1140/epjc/s10052-016-4439-4

6. A.A. Starobinsky, Phys. Lett. B 91, 99 (1980). doi:10.1016/ 0370-2693(80)90670-X

7. P.J. Steinhardt, N. Turok, Science 312, 1180 (2006). doi:10.1126/ science. 1126231

8. G. Allemandi, A. Borowiec, M. Francaviglia, Phys. Rev. D 70, 103503 (2004). doi:10.1103/PhysRevD.70.103503

9. G.J. Olmo, Int. J. Mod. Phys. D 20, 413 (2011). doi:10.1142/ S0218271811018925

10. T. Koivisto, Class. Quantum Gravity 23, 4289 (2006). doi:10.1088/ 0264-9381/23/12/N01

11. A. De Felice, S. Tsujikawa, Living Rev. Relat. 13, 3 (2010). doi:10. 12942/1rr-2010-3

12. T.P. Sotiriou, V. Faraoni, Rev. Mod. Phys. 82, 451 (2010). doi:10. 1103/RevModPhys.82.451

13. S. Capozziello, M.F. De Laurentis, L. Fatibene, M. Ferraris, S. Garruto, SIGMA 12, 006 (2016). doi:10.3842/SIGMA.2016.006

14. G.J. Olmo, Phys. Rev. D 75, 023511 (2007). doi:10.1103/ PhysRevD.75.023511
15. V. Faraoni, Phys. Rev. D 74, 023529 (2006). doi:10.1103/ PhysRevD.74.023529

16. M.P. Dabrowski, J. Garecki, D.B. Blaschke, Ann. Phys. 18, 13 (2009). doi:10.1002/andp.200810331

17. S. Weinberg, Gravitation and Cosmology (Wiley, New York, 1972)

18. J. Beltran Jimenez, L. Heisenberg, G.J. Olmo, JCAP 1506, 026 (2015). doi:10.1088/1475-7516/2015/06/026

19. D. Bazeia, L. Losano, R. Menezes, G.J. Olmo, D. RubieraGarcia, Class. Quantum Gravity 32, 215011 (2015). doi:10.1088/ $0264-9381 / 32 / 21 / 215011$

20. V. Faraoni, E. Gunzig, Int. J. Theor. Phys. 38, 217 (1999). doi:10. 1023/A:1026645510351

21. S. Capozziello, P. Martin-Moruno, C. Rubano, Phys. Lett. B 689, 117 (2010). doi:10.1016/j.physletb.2010.04.058

22. S. Bahamonde, S.D. Odintsov, V.K. Oikonomou, M. Wright, Ann. Phys. 373, 96 (2016). doi:10.1016/j.aop.2016.06.020

23. A. Stachowski, M. Szydlowski, A. Borowiec, Eur. Phys. J. C 77(6), 406 (2017). doi:10.1140/epjc/s10052-017-4981-8

24. C. Bejarano, G.J. Olmo, D. Rubiera-Garcia, Phys. Rev. D 95, 064043 (2017). doi:10.1103/PhysRevD.95.064043

25. C. Bambi, A. Cardenas-Avendano, G.J. Olmo, D. Rubiera-Garcia, Phys. Rev. D 93, 064016 (2016). doi:10.1103/PhysRevD.93. 064016

26. G.J. Olmo, D. Rubiera-Garcia, Universe 1, 173-185 (2015). doi:10. 3390/universe 1020173 